\title{
Iron + folic acid distribution and consumption through antenatal care: identifying barriers across countries
}

\author{
Celeste Sununtnasuk ${ }^{1, *}$, Alexis D'Agostino ${ }^{2}$ and John L Fiedler ${ }^{1}$ \\ 'USAID/SPRING Project (Strengthening Partnerships, Results, and Innovations in Nutrition Globally), Poverty, Health \\ and Nutrition Division, International Food Policy Research Institute, 2033 K St. NW, Washington, DC 20006, USA: \\ ${ }^{2}$ USAID/SPRING Project, JSI Research \& Training Institute, Inc., Rosslyn, VA, USA
}

Submitted 16 June 2014: Final revision received 25 March 2015: Accepted 2 April 2015: First published online 29 May 2015

\begin{abstract}
Objective: The prevalence of maternal anaemia remains unacceptably high in developing countries. At the same time, the percentage of women who consume one or more $\mathrm{Fe}+$ folic acid (IFA) tablets during pregnancy remains persistently low. The objective of the present study was to identify where, within antenatal care (ANC) programmes, pregnant women falter in obtaining and consuming an ideal minimum of 180 IFA tablets.

Design: Data from Demographic and Health Surveys were used to develop a schematic which identifies four sequential 'falter points' to consuming 180 IFA tablets: ANC attendance, IFA receipt or purchase, IFA consumption and the number of tablets consumed.

Setting: Twenty-two countries with high burdens of undernutrition.

Subjects: A sample of 162958 women, 15 to 49 years of age, with a live birth in the past 5 years.

Results: Across all countries, $83 \%$ of all pregnant women had at least one ANC visit, $81 \%$ of whom received IFA tablets. Of those receiving IFA tablets, $95 \%$ consumed at least one. Overall adherence to the ideal supplementation regimen, however, was extremely low: only $8 \%$ consumed 180 or more IFA tablets. There were only two countries in which the percentage of pregnant women consuming 180 or more tablets exceeded $30 \%$.

Conclusions: While most women receive and take some IFA tablets, few receive or take enough. The analysis identifies where ANC-based distribution of IFA falters in each country. It enables policy makers to design and prioritize follow-up activities to more precisely identify barriers, an essential next step to improving IFA distribution through ANC.
\end{abstract}

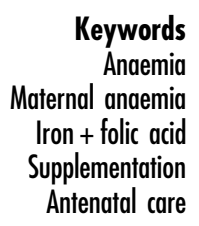

In 2011, after the prevalence of anaemia among pregnant and non-pregnant women had fallen an average of 0.3 percentage points a year since $1995^{(1)}, 528$ million women of childbearing age were anaemic ${ }^{(2)}$. At the current rate of progress, it will be 2043 before 'only' one in three pregnant women and 'only' one in four non-pregnant women are anaemic. The prevalence of anaemia is far too high and progress in reducing it has been far too slow. In 2012, participants in the Sixty-fifth World Health Assembly pledged to reduce the global prevalence of anaemia in women of reproductive age by $50 \%$ by $2025^{(3)}$. Achieving that laudable goal will require tripling the current pace of progress. How might that be accomplished?

Reflecting anaemia's multifaceted nature, WHO guidelines for its prevention and control recommend a cross-sectoral and integrated approach based on its specific causes in a given setting and sub-population ${ }^{(4)}$. Programmes to control and prevent anaemia often involve multiple public and private partners and multiple sectors, such as agriculture to produce more Fe-rich foods, the commercial sector to make more $\mathrm{Fe}$ - and multiple micronutrient-fortified foods available, and the health sector to ensure that women and children receive appropriate services. Appropriate services may include bednets, intermittent preventive treatment and residual household spraying to control helminths and malaria, as well as Fe and multiple micronutrient supplements.

In most countries, two considerations suggest that Fe supplementation programmes will be part of the solution. First, roughly half of the episodes of anaemia that women of childbearing age suffer can be corrected by Fe supplementation $^{(5)}$. Second, globally the most common 
micronutrient supplementation programme implemented at national scale is Fe supplementation for pregnant or non-pregnant women ${ }^{(6)}$. In most low- and middle-income countries, the distribution of $\mathrm{Fe}$ or $\mathrm{Fe}+$ folic acid (IFA) tablets through antenatal care (ANC) was the first national anaemia programme - generally begun three or four decades ago - and it remains the centrepiece of public health anaemia prevention and control efforts. These considerations, plus the growing rates of antenatal coverage that have been achieved in many countries over the past decade, underscore the allure of ANC-based IFA distribution programmes as an indispensable part of a strategy for achieving the World Health Assembly's 2025 goal.

While many countries have had ANC-based IFA distribution programmes for decades, the coverage rates of all but a handful of these programmes are strikingly low. Why? How might their implementation be improved and their impact enhanced? This is an opportune time for reviewing the performance of ANC-based IFA distribution programmes, while keeping in mind that IFA supplementation (and this particular distribution mechanism) should constitute only a part of the strategy to prevent and control anaemia.

Improving the delivery of IFA supplementation through ANC requires understanding how the ANC programme functions within the context of the health-care system ${ }^{(7)}$. An ANC programme that is part of a health-care system that is well-staffed with personnel who have received good training, has good managers and is adequately financed, is likely to function more effectively than one that is lacking in one or more of these dimensions. An adequate supply of IFA requires a logistics system that provides reliably adequate supplies of IFA tablets at national, regional, district and local levels. But, while insufficient IFA tablets is generally regarded as the major supply-side barrier ${ }^{(8-10)}$, an adequate supply of tablets, alone, does not guarantee that IFA tablet distribution coverage will be adequate. Other characteristics of the health-care system, or more specifically of an ANC programme, that are likely to affect IFA tablet distribution include whether or not ANC is provided at a convenient location, at a convenient time of day and for sufficient days during the week to ensure the services are accessible to their target clientele. These structural and organizational supply-side factors influence demand and thereby affect the coverage of the programme.

Even if these conditions are met, however, an adequate supply of IFA does not guarantee that the provider will make them available to the patient; nor does it guarantee that the provider will adequately motivate patient compliance. Given the characteristics of the health-care delivery system, a provider has considerable latitude in choosing what to do. Technical knowledge is but one determinant of how care is provided. Other factors particularly important in the case of IFA supplementation are that providers be adequately motivated to effectively counsel women on the benefits of IFA, that they communicate its potential side-effects and that they adequately follow up to attenuate side-effects and monitor compliance.

From the demand or patient perspective, necessary but not sufficient conditions for the use of any particular anaemia-related programme are that: (i) the service is provided; (ii) is culturally, economically, geographically and socially accessible and (iii) acceptable; and that (iv) the service is valued by the people it is intended to serve. At the individual level on the demand side, people's care-seeking behaviours vary enormously: they are a reflection of a person's predisposition towards using care, enabling factors affecting his/her ability to acquire care and his/her self-perceived need for care. The numerous individual demand-side challenges that play a critical role in affecting the consumption of IFA tablets include: understanding of the significance of Fe-deficiency anaemia; IFA tablet characteristics (e.g. taste, appearance or packaging); women not seeking ANC, seeking it late in their pregnancy or obtaining too few visits; and women's beliefs about actual or possible side-effects and their willingness and ability to effectively communicate with providers ${ }^{(11)}$. Consequently, data about the use of any specific anaemia-related programme are the product of the interaction of a combination of characteristics of both the supply side and the demand side, and it is the outcome of both characteristics of the health system, as well as those of the individual health-care provider and the individual would-be patient. These various factors interact with one another and confound efforts to disentangle the significance of single determinants in trying to assess ANC coverage and utilization rates.

The current paper presents a new approach to analysing both supply and demand factors that condition the success of national efforts to distribute IFA through the ANC system. We review twenty-two countries using a 'falter point' schematic, developed by one of the authors (J.L.F.), which analyses the strengths and weaknesses of the distribution and consumption of IFA supplements through ANC programmes using Demographic and Health Survey (DHS) data. Each falter point aids in identifying where the distribution of IFA through ANC is weak, compromising progress in reducing maternal anaemia. Given the World Health Assembly challenge, there no longer is time for compromising progress. The present analysis can help identify how to systematically begin improving the performance of what is currently the largest programme with the greatest potential for helping to achieve the World Health Assembly goal for 2025.

\section{Data and methodology}

Data on IFA programme coverage, particularly nationwide data, are often non-existent. Monitoring of Fe supplementation programmes is more difficult and less developed than for other micronutrient interventions, due in part to the reliance on regular visits by pregnant women to ANC clinics 
Table 1 Characteristics of DHS used for analysis

\begin{tabular}{lcccc}
\hline Country & Year & $\begin{array}{c}\text { No. of women pregnant in } \\
\text { last 5 years }\end{array}$ & $\begin{array}{c}\% \text { of women pregnant in last } \\
\text { 5 years missing ANC data }\end{array}$ & $\begin{array}{c}\% \text { of women pregnant in last } 5 \text { years } \\
\text { missing IFA consumption data }\end{array}$ \\
\hline Benin & 2006 & 10602 & 1 & 20 \\
Cambodia & 2010 & 6448 & 0 & 15 \\
DRC & 2007 & 5481 & 4 & 58 \\
Ethiopia & 2011 & 7764 & 0 & 79 \\
Ghana & 2008 & 2147 & 3 & 23 \\
Haiti & 2012 & 5414 & 0 & 28 \\
Honduras & 2011 & 8715 & 0 & 42 \\
Indonesia & 2007 & 15334 & 1 & 34 \\
Kenya & 2008 & 4081 & 2 & 43 \\
Liberia & 2007 & 3996 & 12 & 36 \\
Madagascar & 2008 & 8569 & 1 & 44 \\
Malawi & 2010 & 13776 & 1 & 12 \\
Mali & 2006 & 9035 & 2 & 46 \\
Nepal & 2011 & 4079 & 0 & 20 \\
Niger & 2006 & 5884 & 0 & 50 \\
Nigeria & 2008 & 18028 & 8 & 60 \\
Philippines & 2008 & 4712 & 0 & 20 \\
Rwanda & 2010 & 6328 & 0 & 29 \\
Senegal & 2011 & 8151 & 2 & 15 \\
Tanzania & 2010 & 5357 & 1 & 37 \\
Uganda & 2011 & 4909 & 2 & 30 \\
Zambia & 2007 & 4148 & 1 & 15 \\
\hline
\end{tabular}

DHS, Demographic and Health Survey; ANC, antenatal care; IFA, Fe + folic acid; DRC, Democratic Republic of the Congo.

or other distribution centres and the difficulty in measuring the consumption of supplements that must be taken daily. Attempts at estimating coverage are consequently often made indirectly using the number of ANC visits and assuming full compliance; i.e. the compliance of providers with IFA distribution protocols and the compliance of patients with IFA consumption protocols. Given the lack of monitoring capacity and coverage data for Fe supplementation programmes, our contribution in the current paper is to provide a falter point schematic that uses widely available, population-based DHS data to elicit information on the strengths and weaknesses of ANC-based IFA distribution. DHS are nationally representative surveys conducted in over ninety countries that provide comparable statistics on various aspects of health and nutritional status and related behaviours through comprehensive household and individual interviews. The twenty-two countries represented in the present study include those that had a DHS completed during or after 2005 (what were, at the start of the study, the most recently available DHS) and contained information on ANC attendance and IFA consumption. The selection process prioritized countries with high burdens of undernutrition and those of high interest to the US Agency for International Development (USAID), primarily the US Bureau for Food Security's Feed the Future initiative. The countries selected (and the years in which their respective surveys were conducted) are listed in Table 1, with descriptive statistics of the surveys.*

*The majority of the countries included in the present study are discussed in further detail in individual country briefs (A Rapid Initial Assessment of the Distribution and Consumption of Iron Folic Acid Tablets through Antenatal Care, 2014, SPRING Technical Briefs Series).
DHS data have three key limitations. First, due to the intermittent nature of data collection (once every 5 years), the most recent results for countries may not reflect the current situation in the country. As analysis was restricted to countries having DHS that took place after 2005, some of the data illustrated in the figures and discussion may reflect a situation that has changed in the past 10 years. We acknowledge that further research will be needed in many of the areas highlighted in the discussion. Second, there is an absence of supply chain information, which plays such a pivotal role in any ANC system since supply is a necessary but not sufficient condition for compliance. Depending upon where a woman falters, the absence of supply chain information often hinders our ability to draw definitive conclusions regarding the causes of noncompliance. Third, there is potential for recall bias, as ANC and IFA data rely on mother's recall of events occurring up to 5 years before the survey.

Three questions from the DHS, asked of women aged 15-49 years with a live birth in the previous 5 years, were used for analysis in the falter point schematic: (i) 'Did you see anyone for antenatal care for this pregnancy?' (ii) 'During this pregnancy, were you given or did you buy any iron tablets or iron syrup?' (iii) 'During the whole pregnancy, for how many days did you take the tablets or syrup?' For women who reported multiple births in the previous 5 years, data on the most recent birth were used.

Phrasing of the Fe supplementation questions varied slightly across the twenty-two DHS. Only four surveys specify 'iron/folic acid' or 'iron/folate' tablets in the IFA questions, while the remaining eighteen surveys use the more ambiguous terms 'iron tablets' or 'iron syrup'. 
While IFA tablets are typically provided, we recognize that there may be some variance in tablet composition across countries. Women who responded 'yes' to receiving 'iron tablets' or 'iron syrup' during pregnancy may have received a folic acid supplement, either as a separate tablet or combined with $\mathrm{Fe}$ in an IFA tablet. It is possible, however, that our estimates of IFA coverage are higher than 'true' coverage levels if some women are receiving Fe-only supplements.

The Guide to DHS Statistics ${ }^{(12)}$ was used for guidance on the use of weights to properly account for the sampling strategy. All surveys use a stratified two- or three-stage design of sample selection, with stratification by geographic areas and residency type (rural/urban). With the exception of the 2007 Indonesia DHS which included only ever-married women aged 15-49 years, the target populations for the ANC- and IFA-related questions included all women 15-49 years of age. Respondents were read an informed consent statement and were able to choose whether or not to participate. Survey staff also followed strict privacy standards throughout data collection and processing to maintain confidentiality of the respondent's identity and information. All surveys were approved by the ICF Institutional Review Board, as well as in-country institutional review boards, as required.

\section{Falter point schematic}

The falter point schematic provides a new framework for analysing three DHS questions regarding women's receipt and consumption of IFA tablets during pregnancy. Four critical points at which the ANC system might falter in providing IFA are identified: (i) ANC attendance; (ii) IFA receipt or purchase; (iii) IFA consumption; and (iv) compliance with WHO recommendations for the ideal minimum number of tablets. Each sequential decision point represents a potential falter point for pregnant women to consume the ideal minimum of 180 tablets. The falter point schematic is outlined in Fig. 1.

\section{Falter Point 1: ANC attendance}

The first falter point assesses whether or not pregnant women attended ANC, and is calculated as the percentage of women with a live birth in the last 5 years who did not attend at least one ANC visit during their most recent pregnancy. Non-responses were categorized as missing data and not included in calculation of the indicator.

\section{Falter Point 2: IFA receipt or purchase}

At the second falter point, the schematic identifies the percentage of women who attended ANC but did not receive or purchase at least one IFA tablet during their pregnancy. Women who reported that they 'did not know' were categorized as not having received or purchased any tablets during the pregnancy.

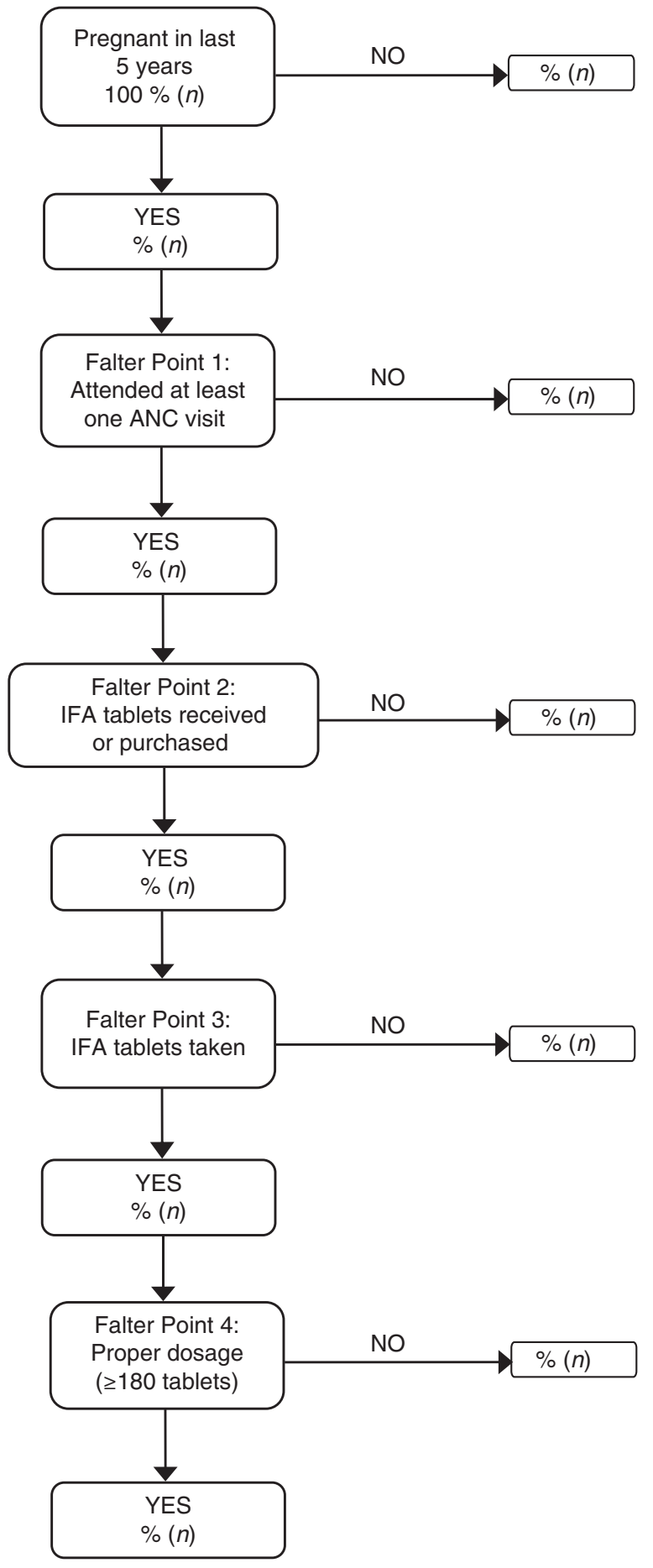

Fig. 1 Falter point schematic to identify four sequential points at which pregnant women may falter in consuming the ideal minimum of 180 IFA tablets (IFA, Fe + folic acid; ANC, antenatal care)

\section{Falter Point 3: Consumption of IFA}

The third falter point identifies the percentage of women who attended ANC and received or purchased one or more IFA tablets, but did not consume at least one tablet. Women who reported that they could not remember how many tablets they took were counted as having consumed no tablets during their pregnancy. 
Falter Point 4: Adberence to $180 \mathrm{~d}$ of supplementation

The final falter point identifies the percentage of women who attended ANC, purchased or received IFA and took at least one tablet, but did not consume the ideal supplementation regimen of 180 or more tablets. As with Falter Point 3, women who reported that they could not remember how many tablets they consumed were counted as having taken no tablets during their pregnancy.

Current WHO guidelines ${ }^{(13)}$ recommend daily IFA supplementation for women throughout pregnancy, beginning as early as possible. Ideally, women should receive IFA tablets no later than the first trimester of pregnancy, corresponding to 6 months (180 d) of IFA supplementation before delivery. Very few women, however, attain these levels of intake. We therefore analyse the percentage of women who consume forty-five, ninety and 135 tablets. Proper consumption of supplements may further be dependent upon the number of ANC visits. Pregnant women must not only seek ANC within the first trimester of pregnancy, but must make multiple visits to obtain the recommended number. We further our analysis by assessing the number of tablets consumed by the number of ANC visits during pregnancy.

\section{Results}

Individual country performances at each of the sequential falter points are presented in Table 2. Performance at Falter Point 1 is particularly low in Ethiopia, Niger and Nigeria, where more than $40 \%$ of all pregnant women did not attend ANC. Half of the reviewed countries (Ghana, Honduras, Indonesia, Liberia, Malawi, the Philippines, Rwanda, Senegal, Tanzania, Uganda and Zambia) however had ANC attendance rates above $95 \%$ of the pregnant female population. Benin, Cambodia and Senegal are the top performers at Falter Point 2. More than 95\% of women who attended ANC in these three countries purchased or received IFA. With only $31 \%$ of pregnant women having received ANC and purchased or received IFA tablets, Ethiopia has the lowest performance at Falter Point 2 and is more than 20 percentage points below the next lowest performer, the Democratic Republic of the Congo (DRC; $48 \%$ ). Variation in country performance is less marked at Falter Point 3, where the majority of women consumed at least one purchased or received IFA tablet. In Nigeria, Liberia, Mali and Kenya, between 12 and $17 \%$ of pregnant women who attended ANC and purchased or received IFA did not consume at least one tablet. The percentages are even smaller in Tanzania, the Philippines, Nepal and Honduras, where $2 \%$ or less of women failed to take at least one tablet. Performance at Falter Point 4 is generally low, with fourteen countries reporting that of those women who attended ANC, purchased or received tablets, and took at least one, fewer than $10 \%$ consumed at least
180 tablets during their pregnancy. In Rwanda, Ethiopia, DRC and Tanzania, nearly all women who reached Falter Point 4 consumed fewer than 180 tablets. Senegal and Nepal are the only countries with a faltering rate below $60 \%$.

Table 3 presents an alternative representation of the importance of each of the falter points in preventing women from taking the ideal minimum number of 180 IFA tablets during their pregnancy. The percentages are calculated using the same denominator - the total number of women of reproductive age who had a live birth within the last 5 years - at each falter point. As such, the way in which the falter point percentages are interpreted will differ from those in Table 2, in which percentages are calculated using changing denominators conditional upon the immediately preceding falter point.

Falter Point 1 is of considerable concern in Mali, Nigeria, Niger and Ethiopia, where nearly one-third to more than one-half ( 29 to $57 \%$ ) of all women do not attend ANC during their pregnancy, suggesting that in these countries, ANC is currently an underutilized vehicle for IFA distribution. After receiving ANC, roughly $40 \%$ of all pregnant women in Honduras, Tanzania and DRC do not receive or purchase IFA (Falter Point 2), constituting a missed opportunity for supplementing pregnant women. With less than $10 \%$ of women in any country failing to take at least one IFA tablet, Falter Point 3 is the least significant of the four falter points.

Roughly $50 \%$ of all women do not consume at least 180 IFA tablets during pregnancy. Despite WHO recommendations, Falter Point 4 is the major faltering point for most countries. Only Ethiopia, Nigeria, Niger, Nepal and DRC have fewer than $40 \%$ of women faltering here; however, this does not mean that a large percentage of pregnant women in these countries are adhering to at least 6 months of supplementation. It is important to bear in mind that for many countries, the low percentage of women faltering at Falter Point 4 is due to high levels of faltering at points 1 and 2. Ethiopia, for example, has the lowest percentage of women faltering at Falter Point 4 (13\%), yet $57 \%$ of women in the country faltered at Falter Point 1; the highest percentage among all twenty-two countries. Taking this into consideration, with the exception of Nepal and Senegal, less than $20 \%$ of women in all countries do not falter (i.e. they have attended ANC, purchased or received tablets, and taken more than 180). Nepal is the overall highest performer, with $38 \%$ of women taking the complete dosage of IFA tablets, followed by Senegal, where $36 \%$ of women took the full dosage.

Achieving $180 \mathrm{~d}$ of supplementation has been a challenge for all twenty-two countries. Table 4 shows that even if the recommended duration of supplementation were shortened to 135,90 or $45 \mathrm{~d}$, many countries would still struggle to meet the recommendations. Among the lowest performing countries in which no more than $2 \%$ of women took 180 or more IFA tablets, less than $15 \%$ of 
Table 2 Percentage of pregnant women faltering at each sequential falter point to consuming an ideal minimum of 180 IFA tablets among a sample of 162958 women (aged 15-49 years with a live birth in the past 5 years) from twenty-two countries that had a DHS completed during or after 2005

\begin{tabular}{|c|c|c|c|c|c|c|c|c|}
\hline \multirow[b]{2}{*}{ Country } & \multicolumn{2}{|c|}{$\begin{array}{l}\text { FP1: pregnant } \\
\text { women who did not } \\
\text { attend ANC }\end{array}$} & \multicolumn{2}{|c|}{$\begin{array}{l}\text { FP2: pregnant women who } \\
\text { passed FP1 but did not } \\
\text { purchase/receive IFA tablets* }\end{array}$} & \multicolumn{2}{|c|}{$\begin{array}{l}\text { FP3: pregnant women who } \\
\text { passed FP1-2 but did not take } \\
\text { at least one IFA tablet* }\end{array}$} & \multicolumn{2}{|c|}{$\begin{array}{c}\text { FP4: pregnant women who } \\
\text { passed FP1- } 3 \text { but did not take } \\
\geq 180 \text { IFA tablets* }\end{array}$} \\
\hline & $\%$ & $95 \% \mathrm{Cl}$ & $\%$ & $95 \% \mathrm{Cl}$ & $\%$ & $95 \% \mathrm{Cl}$ & $\%$ & $95 \% \mathrm{Cl}$ \\
\hline Benin & 11 & 10,13 & 3 & 3,4 & 6 & 5,8 & 77 & 76,79 \\
\hline Cambodia & 10 & 9,12 & 4 & 3,4 & 3 & 2,3 & 98 & 98,99 \\
\hline DRC & 13 & 10,16 & 48 & 43,52 & 10 & 8,13 & 100 & 99,100 \\
\hline Ethiopia & 57 & 54,60 & 69 & 66,71 & 4 & 3,6 & 100 & 99,100 \\
\hline Ghana & 4 & 3,5 & 11 & 10,13 & 6 & 5,8 & 79 & 76,82 \\
\hline Haiti & 9 & 8,11 & 17 & 15,19 & 4 & 3,6 & 88 & 86,89 \\
\hline Honduras & 3 & 3,4 & 40 & 39,42 & 1 & 1,1 & 73 & 71,75 \\
\hline Indonesia & 4 & 4,5 & 19 & 18,21 & 8 & 7,9 & 79 & 77,80 \\
\hline Kenya & 7 & 6,9 & 27 & 24,29 & 12 & 10,14 & 100 & 99,100 \\
\hline Liberia & 4 & 3,5 & 10 & 8,12 & 12 & 10,15 & 99 & 98,99 \\
\hline Madagascar & 9 & 8,10 & 35 & 33,37 & 3 & 3,4 & 98 & 98,99 \\
\hline Malawi & 2 & 1,2 & 7 & 7,8 & 4 & 4,5 & 98 & 98,98 \\
\hline Mali & 29 & 26,32 & 18 & 16,20 & 12 & 10,15 & 93 & 92,94 \\
\hline Nepal & 15 & 13,18 & 9 & 7,11 & 2 & 1,2 & 50 & 47,53 \\
\hline Niger & 53 & 49,57 & 15 & 13,17 & 4 & 3,5 & 98 & 97,99 \\
\hline Nigeria & 40 & 37,43 & 17 & 16,19 & 17 & 16,19 & 91 & 90,93 \\
\hline Philippines & 4 & 3,5 & 15 & 14,17 & 2 & 1,2 & 81 & 79,82 \\
\hline Rwanda & 2 & 1,2 & 26 & 24,27 & 3 & 3,4 & 100 & 100,100 \\
\hline Senegal & 4 & 3,5 & 3 & 2,3 & 9 & 7,11 & 58 & 55,61 \\
\hline Tanzania & 2 & 1,3 & 40 & 37,43 & 2 & 1,3 & 100 & 99,100 \\
\hline Uganda & 4 & 4,5 & 23 & 21,25 & 9 & 8,11 & 99 & 98,99 \\
\hline Zambia & 2 & 2,3 & 8 & 6,9 & 6 & 5,8 & 95 & 94,96 \\
\hline Average & 13 & & 21 & & 6 & & 89 & \\
\hline Weighted average $\dagger$ & 17 & & 25 & & 8 & & 88 & \\
\hline
\end{tabular}

FP1-4, Falter Point 1-4; IFA, Fe + folic acid; DHS, Demographic and Health Survey; ANC, antenatal care; DRC, Democratic Republic of the Congo. *Denominators are conditional upon having complied with or fulfilled the immediately preceding falter point.

†Weighted by 2012 population estimates from the US Census Bureau International Data Base ${ }^{(25)}$.

women consumed a minimum of ninety tablets. Nepal and Senegal remain the top performers when reducing the number of IFA tablets to ninety; however, over one-third of pregnant women in the two countries are unable to achieve even this lower minimum. Less than one-quarter of pregnant women in half of the reviewed countries are able to meet the ninety tablet cut-off.

While it is not possible to determine from DHS data whether IFA was received at the health facility (during ANC or otherwise) or if the respondent purchased the tablets independently, rates of IFA receipt or purchase are much higher among attendees of ANC than non-attendees. This holds true across all countries, even among those with low ANC attendance. Eighty per cent of women across all twenty-two countries who attended ANC received or purchased tablets, compared with only $14 \%$ of women who did not attend ANC (data not shown). The percentage of women who received or purchased IFA without attending ANC is generally $6 \%$ or lower with two notable exceptions: Ethiopia and Niger, where respectively $21 \%$ and $12 \%$ of women who received or purchased IFA did so without having received ANC. Furthermore, as shown in Fig. 2, the number of tablets consumed increases with the number of ANC visits among pregnant women in our sample.

\section{Discussion and conclusion}

Assessing progress in the distribution of IFA through ANCbased programmes is difficult - both at the country level and globally. There is often no data on programme coverage, and monitoring and evaluating the receipt and consumption of IFA tablets at a national scale is cumbersome, challenging and uncommon. The falter point schematic presented here is based on DHS data and, as such, can generally only be updated in a given country once every 5 years. While that is a limitation, the strikingly poor performance of most countries suggests that there is a great deal of room for improvement. Given the longstanding high prevalence and severe nature of anaemia in most lower- and middle-income countries, there is an urgent need to begin the work of improving IFA distribution programmes, which, as already noted, is the oldest and largest anaemia programme in most countries. The availability of these DHS data provides individual countries the opportunity to undertake country-specific diagnostics to identify where ANC-based IFA distribution falters, to develop a strategy for improving its performance and to begin this work now.

Countries in which Falter Point 1 is a major stumbling point are likely to confront the most monumental task in 


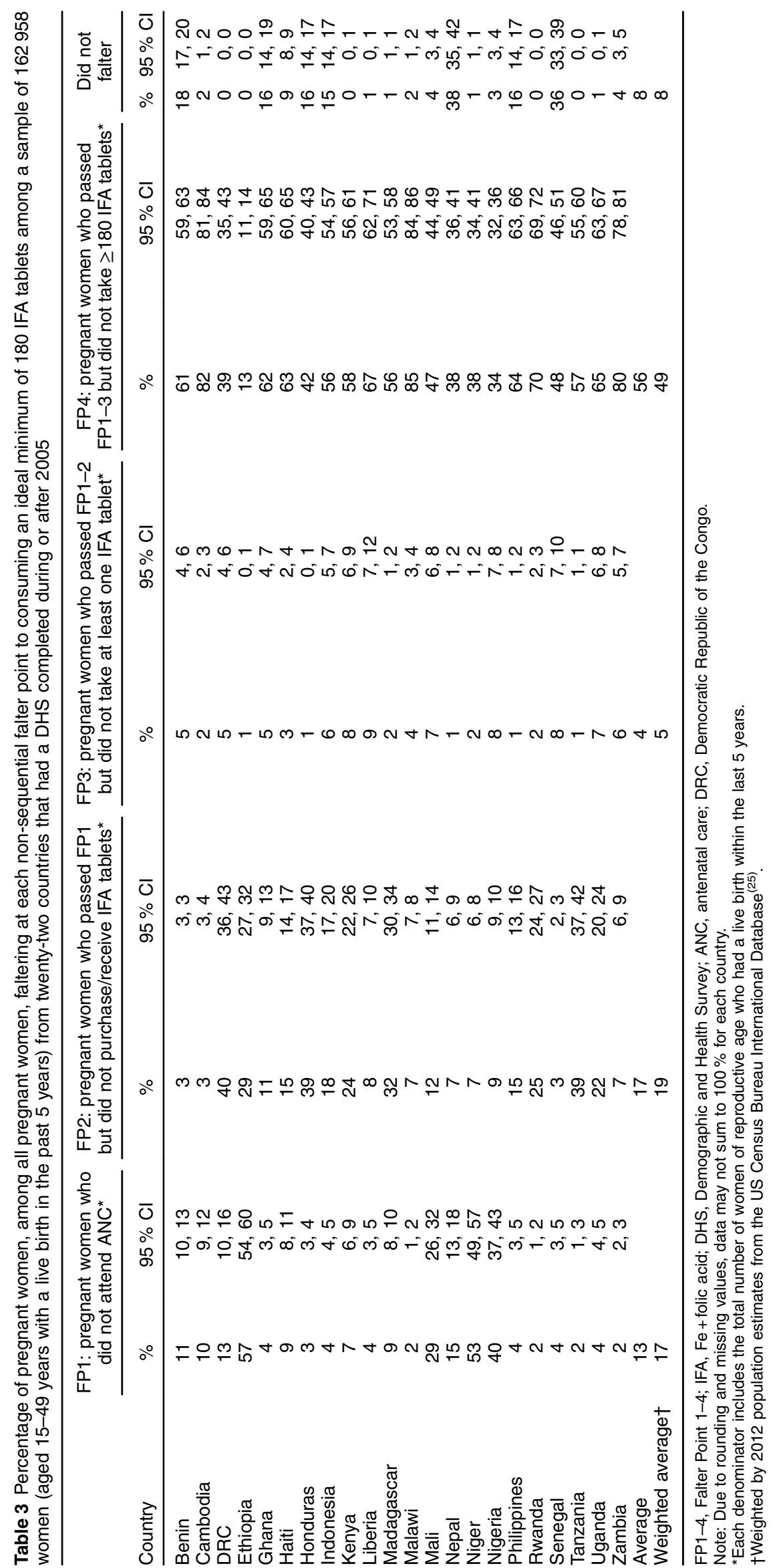


Table 4 Percentage of pregnant women consuming forty-five, ninety, 135 and 180 IFA tablets among a sample of 162958 women (aged 15-49 years with a live birth in the past 5 years) from twenty-two countries that had a DHS completed during or after 2005

\begin{tabular}{|c|c|c|c|c|c|c|c|c|}
\hline \multirow[b]{2}{*}{ Country } & \multicolumn{2}{|c|}{$\geq 45$ IFA tablets } & \multicolumn{2}{|c|}{$\geq 90$ IFA tablets } & \multicolumn{2}{|c|}{$\geq 135$ IFA tablets } & \multicolumn{2}{|c|}{$\geq 180$ IFA tablets } \\
\hline & $\%$ & $95 \% \mathrm{Cl}$ & $\%$ & $95 \% \mathrm{Cl}$ & $\%$ & $95 \% \mathrm{Cl}$ & $\%$ & $95 \% \mathrm{Cl}$ \\
\hline Senegal & 73 & 71,75 & 62 & 60,65 & 45 & 42,48 & 36 & 33,39 \\
\hline Cambodia & 73 & 71,75 & 57 & 54,59 & 3 & 2,4 & 2 & 1,2 \\
\hline Benin & 65 & 63,67 & 53 & 51,55 & 28 & 27,30 & 18 & 17,20 \\
\hline Nepal & 61 & 58,65 & 56 & 52,60 & 44 & 41,48 & 38 & 35,42 \\
\hline Zambia & 59 & 56,62 & 44 & 41,47 & 11 & 10,13 & 4 & 3,5 \\
\hline Ghana & 54 & 50,57 & 41 & 38,45 & 25 & 22,29 & 16 & 14,19 \\
\hline Malawi & 52 & 51,54 & 32 & 31,34 & 4 & 4,5 & 2 & 1,2 \\
\hline Honduras & 48 & 47,50 & 37 & 36,39 & 20 & 19,21 & 16 & 14,17 \\
\hline Philippines & 43 & 41,45 & 34 & 32,36 & 21 & 19,23 & 16 & 14,17 \\
\hline Haiti & 41 & 39,44 & 30 & 27,32 & 13 & 11,14 & 9 & 8,10 \\
\hline Indonesia & 40 & 37,42 & 29 & 27,31 & 19 & 17,20 & 15 & 14,17 \\
\hline Liberia & 33 & 29,38 & 14 & 11,17 & 2 & 2,4 & 1 & 0,1 \\
\hline Mali & 28 & 26,30 & 18 & 17,20 & 6 & 5,7 & 4 & 3,4 \\
\hline Niger & 24 & 21,26 & 14 & 12,16 & 2 & 1,2 & 1 & 1,1 \\
\hline Nigeria & 19 & 17,21 & 14 & 13,16 & 7 & 6,8 & 3 & 3,4 \\
\hline Madagascar & 15 & 14,17 & 8 & 7,9 & 2 & 1,2 & 1 & 1,1 \\
\hline Tanzania & 11 & 9,12 & 3 & 3,4 & 0 & 0,1 & 0 & 0,0 \\
\hline Uganda & 8 & 7,9 & 4 & 3,5 & 1 & 1,2 & 1 & 0,1 \\
\hline Kenya & 6 & 5,7 & 2 & 2,3 & 0 & 0,1 & 0 & 0,1 \\
\hline Rwanda & 4 & 3,4 & 1 & 1,2 & 0 & 0,0 & 0 & 0,0 \\
\hline DRC & 3 & 3,5 & 2 & 1,2 & 0 & 0,1 & 0 & 0,0 \\
\hline Ethiopia & 1 & 1,2 & 0 & 0,1 & 0 & 0,0 & 0 & 0,0 \\
\hline
\end{tabular}

IFA, Fe + folic acid; DHS, Demographic and Health Survey; DRC, Democratic Republic of the Congo.

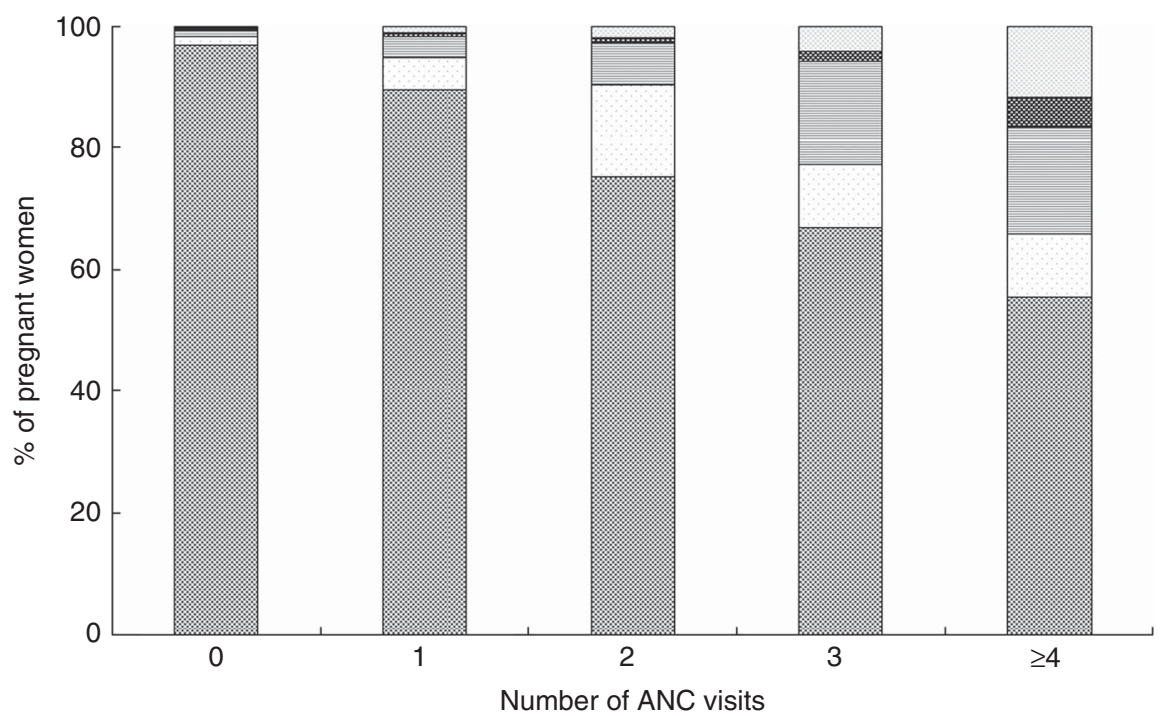

Fig. 2 Percentage of pregnant women consuming IFA tablets ( $\square, \geq 180$; 图, 135-179; 글, 90-135; $\square, 45-89$; 8 , <45) by number of ANC visits among a sample of 162958 women (aged 15-49 years with a live birth in the past 5 years) from twenty-two countries that had a DHS completed during or after 2005 (IFA, Fe + folic acid; ANC, antenatal care; DHS, Demographic and Health Survey)

improving IFA supplementation because it requires strengthening the entire health system at the country level. It involves improving the coverage, acceptability and utilization of the entire system and may entail major cultural change. These are generally long-term propositions. Whether the coverage of ANC is low due to women's lack of physical access to care or because they do not value ANC (for whatever reason), Falter Point 1 manifests a fundamental health system failure. Fortunately, the coverage of ANC has expanded so dramatically globally in the past two decades that Falter Point 1 remains a stumbling block in only a handful of countries.

Countries in which Falter Point 2 is important are likely to have irregular supplies of IFA tablets and may have a more general medical supply problem due to weak logistics systems. Like Falter Point 1, this potential stumbling point is also predominantly a weakness of the supply side of the health-care system. Improving a country's 
performance at Falter Point 2 will initially entail identifying whether a general logistics problem is present, or whether IFA tablets in particular are poorly supplied. The nature of the problem can be better understood by conducting medical supply audits or, more specifically, by tracking the flow of IFA tablets through the system. A number of both qualitative and quantitative tools for analysing the management of logistics systems now exist that would be useful in this work. The Logistics System Assessment Tool (LSAT), developed by one of USAID's longstanding projects, DELIVER, is a qualitative diagnostic and monitoring tool that can be used to provide a comprehensive, systemlevel assessment of a logistics system for any health programme that provides a health commodity. Through qualitative group discussion or key informant interviews, the tool reviews a supply chain's organization and staffing, product selection, forecasting, procurement, warehousing and storage, transport, inventory control and product use $^{(14)}$. The LSAT is commonly used jointly with the Logistics Indicators Assessment Tool (LIAT), also developed by the DELIVER project. While the LSAT provides a comprehensive look at the different components of the logistics system, the LIAT is a quantitative data collection instrument used to assess how well the health commodity logistics system is performing. The LIAT is used to conduct a facilitybased survey to collect information that can be used to calculate indicators related to commodity availability at health facilities (e.g. stocking levels and frequency and duration of stockouts) and determine whether reporting and storage condition standards are being met ${ }^{(15)}$. If conducted annually, over successive years and from a large number of sites, data collected from the LSAT and LIAT can be used to pinpoint a country's system-level strengths and weaknesses and identify areas in which performance needs to be improved. DHS's Service Provision Assessment (SPA) surveys are another tool countries can use to collect information on the availability of facility-based health services and assess whether facilities have the necessary infrastructure, supplies, equipment and conditions to support quality services at the time of the survey. Two of the key services and topics assessed in the SPA which are relevant to this work include (i) infrastructure, resources and systems (including storage and stock monitoring for medicines) and (ii) maternal and newborn health. Summaries of additional instruments that provide health facility-based data and assessments at national and sub-national levels, along with descriptions of their capacity requirements and methodology, are available in a profile of health facility assessment methods published by MEASURE Evaluation ${ }^{(16)}$, as well as on the MEASURE Evaluation website. The exact approach to any of the aforementioned supply-chain-focused assessment activities will vary by country and will depend on the nature of the health-care system and its administrative and managerial systems.

Poor performance at Falter Point 3 may indicate low acceptance of IFA due to ignorance about the importance of Fe during pregnancy, inadequate counselling about Fe and how to manage actual or perceived negative sideeffects. A study of pregnant women in Kenya, for example, found that it was the perceived side-effects of supplements that deterred women from taking the IFA tablets they had received $^{(17)}$. Unwillingness to consume tablets may also be related to factors such as characteristics of the tablets (e.g. colour, form, taste and packaging), cultural beliefs (fear of having a big baby and difficult delivery) and the relationship between the patient and health-care provider ${ }^{(11)}$. More proactive, ANC patient-focused surveys or key informant interviews of ANC providers to identify consumer preferences about tablet characteristics are essential to eschew side-effects, mitigate non-compliance and, in general, improve understanding of the specific causes of non-compliance. Toolkits, training manuals and other materials designed to provide the resources necessary to implement and integrate approaches aimed at improving patient understanding and care among programmes and practitioners are publicly available through the Knowledge for Health (K4HEALTH) Project website.

There are a number of supply- and demand-related factors that affect a country's Falter Point 4 performance. The consumption of 180 tablets requires knowledge of the pregnancy and the importance of IFA supplementation during pregnancy and access to the tablets during the first trimester. If the first ANC visit takes place after the first trimester, it is no longer possible for women to complete the full 180 tablets regimen. Therefore, when women begin their ANC, the obstacles they encounter to early initiation of ANC and the reasons why many of them have fewer than the recommended four visits during a pregnancy are all important issues that need to be understood so that their negative impacts can be mitigated.

Previous WHO guidance on IFA supplementation for pregnant women specified daily supplementation for a minimum of 6 months, the equivalent of 180 tablets $^{(18)}$. As noted earlier, however, many countries do not have explicit national guidelines about the specific number of IFA tablets to be taken. Moreover, some national policies that do address this issue are at variance with WHO recommendations. For instance, Malawi's National Nutrition Policy and Strategic Plan recommends 120 tablets $^{(19)}$. Not all of the countries having guidelines that establish a lower minimum number of days of IFA supplementation than that recommended by WHO, however, are poor performers. While Malawi has a national policy advising fewer than $180 \mathrm{~d}$ of Fe supplementation, more Malawian women consume 180 or more tablets than women in countries whose national guidelines recommend 180 . Some countries' ANC protocols call for dispensing specific quantities of IFA tablets per ANC visit, and often call for distributing only thirty or forty-five tablets. This practice has raised questions about both the motivation for and the impact of this practice on women's ability to achieve taking the recommended minimum of 180 IFA tablets. 
More clear and explicit IFA-related guidance, better documentation and availability of those guidelines, and their more consistent implementation could improve IFA consumption rates.

While improving IFA distribution through ANC systems will be critical, given anaemia's multifactorial aetiology, reducing anaemia will require improving multi-sectoral efforts as well. Although IFA programmes have been important parts of the strategy, the countries that have been more successful in combating anaemia have not worked exclusively through IFA programmes. Nor have they relied exclusively on health facility-based distribution of IFA. Among the most well-known and welldocumented success stories are Nicaragua's Integrated Anemia Control Strategy (IACS), Thailand's National Anemia Reduction Program and the Iron Intensification Project of Nepal (the latter being the top performer among the twenty-two countries analysed here). A key component of those programmes is the community-based approach through which community health workers provided 'near-peer' IFA distribution sites, as well as counselling and follow-up appointments ${ }^{(20-24)}$. Additional factors in the success of these programmes have been their use of IFA as a preventive measure and their use of presumptive treatment; i.e. the provision of supplements regardless of whether or not the woman is currently anaemic.

The urgency of Ayoya et al.'s ${ }^{(10)}$ plea to reify the battle against maternal anaemia is appropriate and applicable well beyond their paper's West Africa focus. It is relevant to the policy makers in most of the twenty-two countries analysed here and others, who likewise are grappling with unacceptably high and stagnant rates of anaemia. Effectively addressing this longstanding problem will require renewed, intensified and sustained commitment at the community, regional and national levels. Achieving the World Health Assembly's 2025 goal of halving maternal anaemia will require multiple programmes and a multisectoral approach. As part of that effort, the renewal efforts to increase the distribution of IFA through ANC is already past due.

\section{Acknowledgements}

Acknowledgements: The authors would like to acknowledge Manisha Tharaney for providing valuable feedback and input on an earlier version of this manuscript. Financial support: This work was supported by the Strengthening Partnerships, Results and Innovations in Nutrition Globally (SPRING) Project of the US Agency for International Development (USAID) under Cooperative Agreement No. AID-OAA-A-11-00031. The funder had no role in the design, analysis or writing of this article. Conflict of interest: None. Authorship: C.S., analysis, report writing. A.D., data processing, analysis, report writing. J.L.F., conceptualization, analysis, report writing. Ethics of buman subject participation: All DHS were approved by the ICF Institutional Review Board, as well as in-country institutional review boards, as required.

\section{References}

1. Mason J, Martorell R, Saldanha L et al. (2013) Reduction in anaemia. Lancet Glob Health 1, e4-e6.

2. Stevens GA, Finucane MM, De-Regil LM et al. (2013) Global, regional, and national trends in haemoglobin concentration and prevalence of total and severe anaemia in children and pregnant and non-pregnant women for 1995-2011: a systematic analysis of population-representative data. Lancet Glob Health 1, e16-e25.

3. Branca F, Mahy L \& Mustafa TS (2014) The lack of progress in reducing anaemia among women: the inconvenient truth. Bull World Health Organ 92, 231.

4. World Health Organization (2001) Iron Deficiency Anaemia. Assessment, Prevention and Control. A Guide for Program Managers. Geneva: WHO.

5. Black RE, Victora CG, Walker SP et al. (2013) Maternal and child undernutrition and overweight in low-income and middle-income countries. Lancet 382, 427-451.

6. World Health Organization (2013) Global Nutrition Policy Review: What Does it Take to Scale up Nutrition? Geneva: WHO.

7. Mwadime RKN, Harvey PWJ, Naikoba S et al. (2002) Overcoming Barriers to Effective Maternal Anemia Interventions during Antenatal Services in Uganda. Kampala: Regional Centre for Quality of Health Care (RCQHC) and the MOST Project.

8. Galloway R, Dusch E, Elder L et al. (2002) Women's perceptions of iron deficiency and anemia prevention and control in eight developing countries. Soc Sci Med 55, 529-544.

9. Miaffo C, Some F, Kouyate B et al. (2004) Malaria and anemia prevention in pregnant women of rural Burkina Faso. BMC Pregnancy Childbirth 4, 18.

10. Ayoya MA, Bendech MA, Zagré NM et al. (2012) Maternal anaemia in West and Central Africa: time for urgent action. Public Health Nutr 15, 916-927.

11. Galloway R \& McGuire J (1994) Determinants of compliance with iron supplementation: supplies, side effects, or psychology? Soc Sci Med 39, 381-390.

12. Rutstein SO \& Rojas G (2006) Guide to DHS Statistics. Demographic and Health Surveys Methodology. Calverton, MD: ORC Macro.

13. World Health Organization (2012) Guideline: Daily Iron and Folic Acid Supplementation in Pregnant Women. Geneva: WHO.

14. US Agency for International Development (2009) Logistics System Assessment Tool (LSAT). Arlington, VA: USAID । DELIVER Project.

15. US Agency for International Development (2011) Guide to Conducting Supply Chain Assessments Using the LSAT and LIAT. Arlington, VA: USAID I DELIVER Project, Task Order 1.

16. Hozumi D, Fronczak N, Minichiello NS et al. (2008) Profiles of Health Facility Assessment Methods (TR-06-36). Chapel Hill, NC: MEASURE Evaluation.

17. Maina-Gathigi L, Omolo J, Wanzala P et al. (2013) Utilization of folic acid and iron supplementation services by pregnant women attending an antenatal clinic at a regional referral hospital in Kenya. Matern Child Health J 17, 1236-1242.

18. World Health Organization (2006) Iron and Folate Supplementation. Standards for Maternal and Neonatal Care, Integrated Management of Pregnancy and Childbirth (IMPAC) Vol. 1.8. Geneva: WHO.

19. Office of the President and Cabinet (2008) Infant and Young Child National Nutrition Policy and Strategic Plan. Lilongwe: Government of Malawi. 
20. Mora JO (2007) Integrated Anemia Control Strategy has Significantly Reduced Anemia in Women and Children in Nicaragua. Ottawa: Micronutrient Initiative.

21. Winichagoon P (2002) Prevention and control of anemia: Thailand experiences. J Nutr 132, Suppl. 4, 862S-866S.

22. Maternal and Child Health Integrated Program (2011) Community-Based Distribution for Routine Iron/Folic Acid Supplementation in Pregnancy. http://www.mchip.net/ sites/default/files/MCHIP\%20Brief_community\%20IFA.pdf (accessed April 2014).
23. Sanghvi TG, Harvey PWJ \& Wainwright E (2010) Maternal iron-folic acid supplementation programs: evidence of impact and implementation. Food Nutr Bull 31, Suppl. 2, S100-S107.

24. Pokharel RJ, Maharjan MR, Mathema P et al. (2011) Success in Delivering Interventions to Reduce Maternal Anemia in Nepal: $A$ Case Study of the Intensification of Maternal and Neonatal Micronutrient Program. Washington, DC: A2Z Project, USAID.

25. US Census Bureau (2012) International Data Base. http:// www.census.gov/population/international/data/idb/inform ationGateway.php (accessed August 2012). 